PROCEEDINGS OF THE

AMERICAN MATHEMATICAL SOCIETY

Volume 135, Number 7, July 2007, Pages 2127-2130

S 0002-9939(07)08722-9

Article electronically published on March 2, 2007

\title{
A NOTE ON INTERPOLATION BY BLOCH FUNCTIONS
}

\author{
DANIEL PASCUAS
}

(Communicated by Juha M. Heinonen)

\begin{abstract}
We show that the positive part of a result on interpolation by Bloch functions due to B. Bøe and A. Nicolau is a direct consequence of C. Sundberg's description of the traces of BMOA functions on interpolating sequences for $H^{\infty}$.
\end{abstract}

The Bloch space $\mathcal{B}$ consists of all the analytic functions $f$ in the unit disk $\mathbb{D}$ of the complex plane such that

$$
\|f\|_{\mathcal{B}}=\sup _{z \in \mathbb{D}}\left(1-|z|^{2}\right)\left|f^{\prime}(z)\right|<\infty .
$$

Recall that the hyperbolic distance between two points $z, w \in \mathbb{D}$ is

$$
d(z, w)=\frac{1}{2} \log \frac{1+\rho(z, w)}{1-\rho(z, w)},
$$

where $\rho(z, w)=|(z-w) /(1-\bar{z} w)|$ is the pseudohyperbolic distance between them. It turns out that an analytic function $f$ in $\mathbb{D}$ belongs to $\mathcal{B}$ if and only if it is a Lipschitz function from $\mathbb{D}$ to $\mathbb{C}$, when $\mathbb{D}$ is equipped with the hyperbolic metric and $\mathbb{C}$ with the Euclidean metric. Indeed,

$$
\|f\|_{\mathcal{B}}=\sup \{|f(z)-f(w)| / d(z, w): z, w \in \mathbb{D}, z \neq w\} .
$$

Thus it seems natural to say that a sequence of points $\left\{z_{j}\right\}_{j \geq 1}$ in $\mathbb{D}$ is interpolating for $\mathcal{B}$ if for any sequence of complex numbers $\left\{\alpha_{j}\right\}_{j \geq 1}$ satisfying the Lipschitz condition $\left|\alpha_{j}-\alpha_{k}\right|=O\left(d\left(z_{j}, z_{k}\right)\right)$ there is some $f \in \mathcal{B}$ such that $f\left(z_{j}\right)=\alpha_{j}$, for every $j \geq 1$. B. Bøe and A. Nicolau in [1] obtained a very nice geometrical characterization of those sequences by means of the following result.

Theorem A. A sequence $\left\{z_{j}\right\}_{j \geq 1}$ in $\mathbb{D}$ is interpolating for $\mathcal{B}$ if and only if it is the union of at most two separated sequences and satisfies the following condition:

(A) There exists a constant $0<\alpha<1$ such that

$$
\#\left\{z_{j}: \rho\left(z_{j}, z\right)<r\right\} \lesssim(1-r)^{-\alpha} \quad(z \in \mathbb{D}, 0<r<1) .
$$

Recall that a sequence $\left\{z_{j}\right\}_{j \geq 1}$ in $\mathbb{D}$ is separated when $\inf _{j \neq k} \rho\left(z_{j}, z_{k}\right)>0$. As usual, the notation $A \lesssim B$ means that $A$ is less than or equal to a constant times

Received by the editors November 30, 2005 and, in revised form, March 16, 2006.

2000 Mathematics Subject Classification. Primary 30E05, 30D45; Secondary 30 D50.

Key words and phrases. Interpolation, Bloch functions, BMOA.

The author was partially supported by the DGI grant MTM2005-08984-C02-02 and the DURSI grant 2005 SGR00611.

(C)2007 American Mathematical Society Reverts to public domain 28 years from publication 
$B$, where the constant is independent of the variables involved (e.g. $z \in \mathbb{D}$ and $0<r<1$, in the statement of Theorem A).

The goal of this note is to give a simple proof of the sufficiency part of Theorem $\mathrm{A}$. In order to motivate it let us review the original proof of that result by Bøe and Nicolau. There are two conditions involved in Theorem $\mathrm{A}$ the separation condition and condition (A). The last one is the most important of the two. Roughly speaking, (A) means that the points in the sequence are exponentially more sparse than they are in a separated sequence, since such a sequence satisfies condition (A) with $\alpha=1$.

The necessity of the separation can be proved along almost the same lines as the corresponding fact for the space of analytic functions in $\mathbb{D}$ which are Lipschitz with respect to the Euclidean metrics (see [5]).

The proof of the necessity of condition (A) has its roots in the seminal work of N. Makarov [6]. The authors proved (A) by combining a simple reproducing formula due to Nicolau [7] and stopping time arguments. They suggested that it also follows from the subgaussian estimates. This alternative proof of (A) is performed in detail by K. Seip in 8 .

On the other hand, a simple argument by Bøe and Nicolau reduces the proof of the sufficiency in Theorem A to the case of separated sequences. Then they show that condition (A) implies that the interpolation problem for $\mathcal{B}$ can be solved by functions in the subspace BMOA of $\mathcal{B}$. That is the hardest part of the proof of Theorem $\mathrm{A}$ It is done in two steps. First, they construct a smooth non-analytic solution $\varphi$ of the interpolation problem such that $|\nabla \varphi(x+i y)| d x d y$ is a Carleson measure. Then they obtain an interpolating function in BMOA by solving a $\bar{\partial}$ problem, using standard techniques. The function $\varphi$ is constructed from its dyadic version by an averaging procedure, originally due to J. Garnett and P. Jones 4], which, as the authors comment, was also used by C. Sundberg in 9 to prove the following beautiful theorem.

Theorem B. Let $\left\{z_{j}\right\}_{j \geq 1}$ be an interpolating sequence for $\mathrm{H}^{\infty}(\mathbb{D})$, and let $\left\{\alpha_{j}\right\}_{j \geq 1}$ be a sequence of complex numbers. Then there is a function $f \in \mathrm{BMOA}$ such that $f\left(z_{j}\right)=\alpha_{j}$, for every $j \geq 1$, if and only if $\left\{\alpha_{j}\right\}_{j \geq 1}$ satisfies the following condition:

(B) There is a constant $\lambda>0$ and a function $\alpha: \mathbb{D} \rightarrow \mathbb{C}$ such that

$$
\sup _{z \in \mathbb{D}} \sum_{j=1}^{\infty} e^{\lambda\left|\alpha_{j}-\alpha(z)\right|}\left(1-\rho\left(z, z_{j}\right)^{2}\right)<\infty .
$$

Recall that $\mathrm{H}^{\infty}=\mathrm{H}^{\infty}(\mathbb{D})$ is the space of bounded analytic functions in $\mathbb{D}$, and a sequence $\left\{z_{j}\right\}_{j \geq 1}$ in $\mathbb{D}$ is interpolating for $\mathrm{H}^{\infty}$ if for every bounded sequence of complex numbers $\left\{\alpha_{j}\right\}_{j \geq 1}$ there is some $f \in \mathrm{H}^{\infty}$ such that $f\left(z_{j}\right)=\alpha_{j}$, for any $j \geq 1$. A classical theorem of L. Carleson [2] characterizes those sequences as the ones which are separated and satisfy the so-called Carleson condition:

$$
\sup _{j \geq 1} \sum_{k=1}^{\infty}\left(1-\rho\left(z_{j}, z_{k}\right)^{2}\right)<\infty .
$$

In the remaining part of this note we are going to show that the sufficiency of Theorem $\mathrm{A}$ is (essentially) an easy consequence of Theorem B Namely, we will prove that Theorem $\mathrm{B}$ implies that a separated sequence which satisfies (A) is interpolating for $\mathcal{B}$. Indeed, the interpolation is performed by functions in BMOA. 
First we obtain an equivalent form of condition (A) which fits better with condition (B).

Lemma. For a sequence $\left\{z_{j}\right\}_{j \geq 1}$ in $\mathbb{D}$, condition (A) is equivalent to

There is a constant $0<\beta<1$ such that

$$
M=\sup _{z \in \mathbb{D}} \sum_{j=1}^{\infty}\left(1-\rho\left(z, z_{j}\right)^{2}\right)^{\beta}<\infty
$$

Proof. Let $n_{z}(r)=\#\left\{z_{j}: \rho\left(z_{j}, z\right)<r\right\}$, for $z \in \mathbb{D}$ and $0<r<1$.

$(\mathrm{A}) \Rightarrow$ (A' $):$ Pick $\beta \in \mathbb{R}$ such that $\alpha<\beta<1$. Then

$$
\sum_{j=1}^{\infty}\left(1-\rho\left(z_{j}, z\right)^{2}\right)^{\beta}=\int_{0}^{1}\left(1-r^{2}\right)^{\beta} d n_{z}(r),
$$

and, taking into account (A), an integration by parts shows $\left(\mathrm{A}^{\prime}\right)$ :

$$
\int_{0}^{1}\left(1-r^{2}\right)^{\beta} d n_{z}(r) \lesssim \int_{0}^{1}\left(1-r^{2}\right)^{\beta-1} n_{z}(r) d r \lesssim \int_{0}^{1} \frac{d r}{(1-r)^{1+\alpha-\beta}}<\infty .
$$

$\underline{\left(\mathrm{A}^{\prime}\right.} \Rightarrow$ : For every $z \in \mathbb{D}$ and $0<r<1$ we have that

$$
(1-r)^{\beta} n_{z}(r) \leq \sum_{j=1}^{\infty}\left(1-\rho\left(z_{j}, z\right)^{2}\right)^{\beta} \leq M
$$

and (A) follows with $\alpha=\beta$.

Now assume $\left\{z_{j}\right\}_{j \geq 1}$ is a separated sequence in $\mathbb{D}$ satisfying condition (A' and let $\left\{\alpha_{j}\right\}_{j \geq 1}$ be a sequence of complex numbers so that

$$
\left|\alpha_{j}-\alpha_{k}\right| \lesssim d\left(z_{j}, z_{k}\right) \quad(1 \leq j<k) .
$$

We want to show that there is an $f \in$ BMOA such that $f\left(z_{j}\right)=\alpha_{j}$, for every $j \geq 1$.

First note that (Amplies (C), so $\left\{z_{j}\right\}_{j \geq 1}$ is interpolating for $\mathrm{H}^{\infty}$. Thus, by Theorem $\mathrm{B}$, we only have to check that the sequences $\left\{z_{j}\right\}_{j \geq 1}$ and $\left\{\alpha_{j}\right\}_{j \geq 1}$ satisfy (B).

A well-known result on extension of Lipschitz functions (see [3, p. 202]) assures the existence of a function $\alpha: \mathbb{D} \rightarrow \mathbb{C}$ such that $\alpha\left(z_{j}\right)=\alpha_{j}$, for every $j \geq 1$, and

$$
|\alpha(z)-\alpha(w)| \lesssim d(z, w) \quad(z, w \in \mathbb{D})
$$

In particular,

$$
\left|\alpha_{j}-\alpha(z)\right|=\left|\alpha\left(z_{j}\right)-\alpha(z)\right| \lesssim d\left(z_{j}, z\right) \quad(j \geq 1, z \in \mathbb{D}) .
$$

Then we may take $\lambda>0$ so small that

$$
e^{\lambda\left|\alpha_{j}-\alpha(z)\right|} \lesssim \frac{1}{\left(1-\rho\left(z_{j}, z\right)^{2}\right)^{1-\beta}} \quad(j \geq 1, z \in \mathbb{D}) .
$$

Therefore $\left(\mathrm{A}^{\prime}\right)$ implies $(\mathrm{B})$, and we are done. 


\section{REFERENCES}

1. Bjarte Bøe and Artur Nicolau, Interpolation by functions in the Bloch space, J. Anal. Math. 94 (2004), 171-194. MR2124459 (2005k:30052)

2. Lennart Carleson, An interpolation problem for bounded analytic functions, Amer. J. Math. 80 (1958), 921-930. MR0117349 (22:8129)

3. Herbert Federer, Geometric measure theory, Die Grundlehren der mathematischen Wissenschaften, Band 153, Springer-Verlag New York Inc., New York, 1969. MR0257325 (41:1976)

4. John B. Garnett and Peter W. Jones, BMO from dyadic BMO, Pacific J. Math. 99 (1982), no. 2, 351-371. MR658065 (85d:42021)

5. Eric P. Kronstadt, Interpolating sequences for functions satisfying a Lipschitz condition, Pacific J. Math. 63 (1976), no. 1, 169-177. MR.0412431 (54:557)

6. N. G. Makarov, Probability methods in the theory of conformal mappings, Algebra i Analiz 1 (1989), no. 1, 3-59; translation in Leningrad Math. J. 1 (1990), no. 1, 1-56. MR1015333 (90k:30008)

7. Artur Nicolau, Radial behaviour of harmonic Bloch functions and their area function, Indiana Univ. Math. J. 48 (1999), no. 4, 1213-1236. MR.1757073 (2001f:42033)

8. Kristian Seip, Interpolation and sampling in spaces of analytic functions, University Lecture Series, vol. 33, American Mathematical Society, Providence, RI, 2004. MR2040080|(2005c:30038)

9. Carl Sundberg, Values of BMOA functions on interpolating sequences, Michigan Math. J. 31 (1984), no. 1, 21-30. MR736465 (85j:46035)

Departament de Matemàtica Aplicada i Anàlisi, Facultat de Matemàtiques, UniverSitat de Barcelona, Gran Via 585, 08071 Barcelona, Spain

E-mail address: daniel_pascuas@ub.edu 